Article

\title{
The Squeezed Light Source for the Advanced Virgo Detector in the Observation Run $\mathrm{O} 3$
}

\author{
Moritz Mehmet $*,+($ i) and Henning Vahlbruch *,+(i) \\ Institut für Gravitationsphysik, Leibniz Universität Hannover and Max-Planck-Institut für Gravitationsphysik \\ (Albert-Einstein-Institut), Callinstr. 38, 30167 Hannover, Germany \\ * Correspondence: moritz.mehmet@aei.mpg.de (M.M.); henning.vahlbruch@aei.mpg.de (H.V.) \\ + These authors contributed equally to this work.
}

Received: 28 October 2020; Accepted: 25 November 2020; Published: 26 November 2020

check for updates

\begin{abstract}
From 1 April 2019 to 27 March 2020, the Advanced Virgo detector, together with the two Advanced LIGO detectors, conducted the third joint scientific observation run O3, aiming for further detections of gravitational wave signals from astrophysical sources. One of the upgrades to the Virgo detector for $\mathrm{O} 3$ was the implementation of the squeezed light technology to improve the detector sensitivity beyond its classical quantum shot noise limit. In this paper, we present a detailed description of the optical setup and performance of the employed squeezed light source. The squeezer was constructed as an independent, stand-alone sub-system operated in air. The generated squeezed states are tailored to exhibit high purity at intermediate squeezing levels in order to significantly reduce the interferometer shot noise level while keeping the correlated enhancement of quantum radiation pressure noise just below the actual remaining technical noise in the Advanced Virgo detector.
\end{abstract}

Keywords: quantum noise; non-classical light; squeezed vacuum states of light; Advanced Virgo gravitational wave detector; scientific run $\mathrm{O} 3$

\section{Introduction}

Almost 40 years after squeezed vacuum states of light were proposed as a means to improve the sensitivity of interferometric gravitational wave (GW) detectors [1], both the Advanced Virgo detector and the Advanced LIGO detectors have been operating with a quantum enhanced sensitivity during their third joint observation run O3 [2,3]. Many years of experimental research with a focus on developing the necessary concepts and technologies for the generation and application of squeezed light had been necessary to turn the conceptual idea of a non-classical sensitivity enhancement into a key technology for achieving today's unprecedented astrophysical reach. This includes, for example, the generation and control of squeezed states in the audio band [4-6], the observation of squeezing levels beyond $10 \mathrm{~dB}$ quantum noise reduction [7-9], proof of concepts at large scale detectors [10,11] and the long term operation of squeezing at the GEO600 detector [12] since 2010. An overview on the application of squeezed light for GW detectors can be found in Refs. [13,14].

The basic idea behind squeezed light enhanced interferometry is that the detector sensitivity can be improved beyond its so-called quantum noise limit via the injection of squeezed states of light into the actual measurement output (dark port) of the interferometer [1]. Although no bright laser light enters the interferometer through its dark port, the quantum fluctuations of the vacuum field of the light do enter the system and are superimposed with the electromagnetic interferometer output field containing the gravitational wave signal. Replacing the vacuum fluctuations by the continuous injection of squeezed vacuum states of light facilitates a manipulation of the measurement uncertainty. This approach has successfully been used to overcome quantum shot noise limited GW detector 
sensitivities $[2,3,10,11]$, and allowed the demonstration of quantum correlations between light and the kilogram-mass detector mirrors $[15,16]$.

The noise of an electromagnetic field can be described by the amplitude quadrature $\hat{X}_{+}$and the phase quadrature $\hat{X}_{-}$. The product of the variances $\left(\Delta^{2}\right)$ of these two quantities is subject to a Heisenberg uncertainty relation of the form $\Delta^{2} \hat{X}_{+} \cdot \Delta^{2} \hat{X}_{-} \geq 1$. The smallest achievable value is 1 and the minimal, symmetrically distributed uncertainty, $\Delta^{2} \hat{X}_{+}=\Delta^{2} \hat{X}_{-}=1$, corresponds to the vacuum noise of the light field. While this uncertainty relation predicts a lower limit for the product, it allows the reduction of the noise in one quadrature if, simultaneously, the noise (i.e., the uncertainty) in the other quadrature increases. A state where the variance in one of the quadratures is less than 1 is called a squeezed state. Similarly, anti-squeezing describes the noise increase in the orthogonal quadrature. Today's most efficient topology for the generation of continuous-wave squeezed states of light is cavity-enhanced parametric down-conversion, also called optical parametric amplification. The interaction between the fundamental field and its second harmonic via a $\chi^{(2)}$-process inside a non-linear crystal produces non-classical photon-pair correlations that yield a reduced noise variance in a certain field quadrature. This approach has been the basis for all squeezed light sources that have been operated in interferometric gravitational wave detectors so far.

The installation of the squeezed light technology at the Advanced Virgo detector to reduce its quantum noise was part of the upgrade phase prior to O3. While the obtained results were reported in [2], we present here a detailed description of the squeezed light source itself. In contrast to the squeezed light source installed at the two LIGO detectors, the squeezer was realized as a portable stand-alone system, which was operated in air on an external optical bench which is located close to the in-vacuum interferometer readout system.

\section{Setup of the Squeezed Light Source}

The concept of designing a squeezed light source as a self-contained subsystem allows maximum independence from the status and infrastructure of the Advanced Virgo detector. Therefore, thorough pre-commissioning and independent construction, operation and characterization of the squeezed light source was possible. The entire squeezed light source is constructed on an optical breadboard with the footprint of $105 \mathrm{~cm} \times 110 \mathrm{~cm}$. For laser safety and protection against dust and air turbulence, the setup is sealed with side panels and two top plates that can cover the device during operation. Figure 1 shows a picture of the system during the assembly phase in a class 100 clean room at the AEI Hannover, Germany.

Only two light fields exit the box through corresponding openings: On one side, the squeezed vacuum states are provided, and on the other side, a tap-off beam from the main squeezer laser can be used to stabilize the squeezed light source to the fundamental interferometer laser frequency by means of an external phase locking loop (PLL).

A schematic of the optical layout is shown in Figure 2. To provide the necessary level of system autonomy and debugging capability, a diagnostic balanced homodyne detector (BHD) and an additional auxiliary coherent control field (Laser 2) are included in addition to the squeezed light generation itself. This allows for three modes of operation:

1. Local alignment and performance check of the squeezed light source.

2. Matching of the squeezed field to the interferometer by means of an auxiliary bright alignment beam (BAB).

3. Injection of squeezed vacuum states into the interferometer.

In the following subsections, we will describe the main building blocks of the squeezed light source in more detail: 


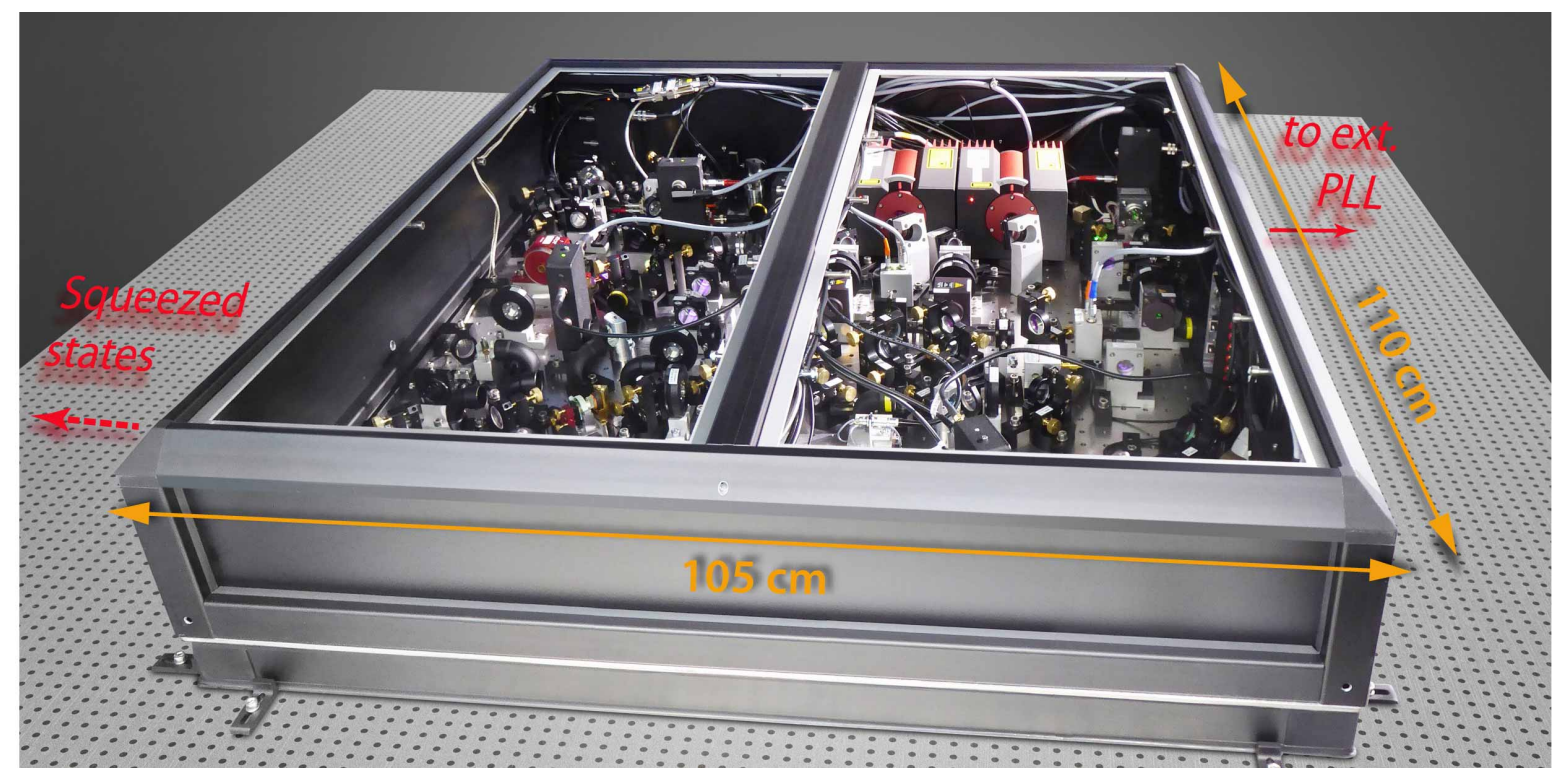

Figure 1. Picture of the squeezed light source on an optical table. The squeezed light source has two output ports to deliver the squeezed states and the tap-off for an external phase locking loop (PLL), respectively.

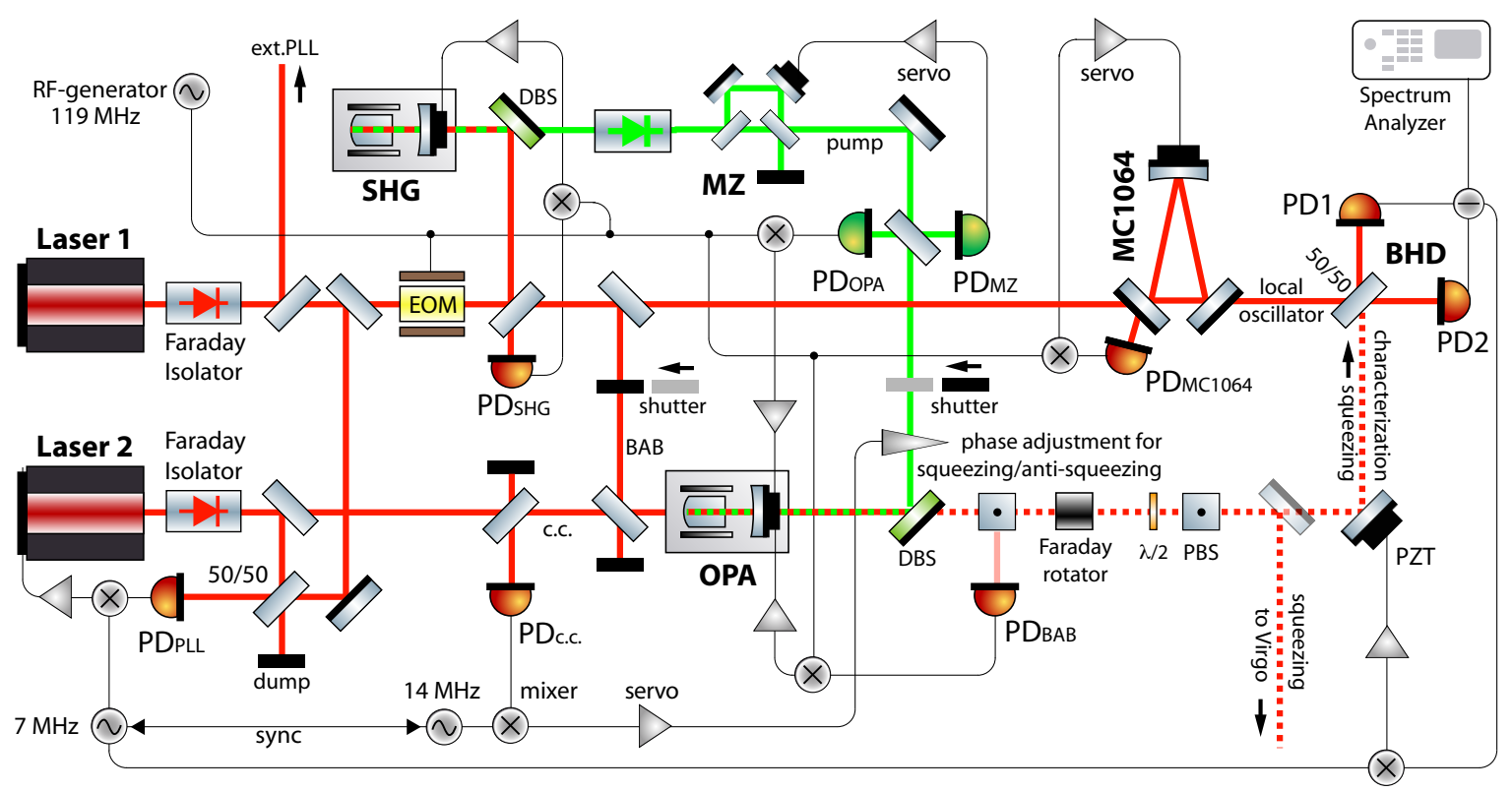

Figure 2. Schematic of the experiment. The generation and the coherent control of squeezed vacuum states of light at a wavelength of $1064 \mathrm{~nm}$ are realized by utilizing two independent but frequency offset locked laser sources. Laser 1 provides the main carrier frequency for homodyne detection and frequency doubling in a second harmonic generator (SHG), which provides the pump field at a wavelength of $532 \mathrm{~nm}$ required for the generation of squeezed vacuum states in an optical parametric amplifier (OPA) operated below threshold. The green pump power is stabilized by a Mach-Zehnder interferometer. The squeezed vacuum states exit the OPA and are separated from the pump field by a dichroic beamsplitter (DBS). A Faraday isolator is installed in the squeezing path. It protects the OPA from backscattered light and suppresses retro-reflections into the interferometer or the BHD. By means of an HR mirror mounted on a motorized translation stage the squeezing is either directed towards the application or onto the balanced homodyne detector (BHD) for characterization. The phase of the green pump, as well as the phase between the squeezed field and the LO, are stabilized by the implementation of a coherent control scheme based on the $7 \mathrm{MHz}$ frequency shifted Laser 2 output field. 


\subsection{Lasers}

The main laser source (Laser 1) is used to drive the second harmonic generator (SHG), required to generate the pump field for the OPA, and provides the bright alignment beam (BAB) and local oscillator field for diagnostic balanced homodyne detection (BHD). Laser 2 is used solely to provide a frequency shifted control field (see below) which allows for the coherent control of the phase of the generated squeezing, either when operating the on-board BHD or when injecting the squeezed states into the gravitational wave detector. Both lasers are non-planar ring-oscillators with single-frequency, continuous-wave output at a wavelength of $1064 \mathrm{~nm}$. They have a maximum output power of $1 \mathrm{~W}$ (Laser 1) and 0.5 W (Laser 2), respectively. Faraday isolators are used to protect the lasers from back-reflected light. A 1\% pick-off from each laser is sent towards a 50/50 beam splitter where the two beams interfere. This generates a beat signal on PDPLL, which serves as the error signal for a phase-locking loop (PLL). Laser 1 serves as the main laser to which Laser 2 is phase locked at a frequency separation of $7 \mathrm{MHz}$ with a control bandwidth of $50 \mathrm{kHz}$. The PLL feedback is applied to Laser 2 by actuating on its laser crystal temperature for compensating long-term drifts of the frequency while a piezo-element attached to the laser crystal is used for fast actuation. A sinusoidal phase modulation at a frequency of $119 \mathrm{MHz}$ is imprinted on the remaining field of Laser 1 by means of an electro-optical modulator (EOM). The phase modulation is used to obtain Pound-Drever-Hall $(\mathrm{PDH})$ error signals for length control of the down-stream cavities.

\subsection{Pump Field Preparation}

A fraction of the light from Laser 1 is used to generate the pump field for the optical parametric amplifier (OPA) at twice the fundamental frequency. The light is mode-matched into a second-harmonic generation (SHG) cavity to generate the $532 \mathrm{~nm}$ field. The SHG is a single-ended standing wave cavity constructed around a $5 \mathrm{~mm}$ long temperature stabilized non-linear crystal made from periodically-poled potassium titanyl phosphate (PPKTP). The optical resonator is formed by the crystal's curved back side and a curved piezo-actuated coupling mirror. The coupling mirror has a transmissivity for the fundamental field of approximately $10 \%$ and has an anti-reflection (AR) coating for the second-harmonic. The crystal back side is highly reflective (HR) for both wavelengths while the plane front-side is AR-coated for both fields to achieve minimal intra-cavity loss. The fundamental and harmonic fields are separated by a dichroic beam splitter (DBS). Demodulating the signal on $\mathrm{PD}_{\mathrm{SHG}}$ in reflection of the SHG yields an error signal which serves as the input to a servo-control loop controlling the position of the piezo-mounted coupling mirror, thereby keeping the SHG on resonance to the fundamental frequency of Laser 1 . The generated $532 \mathrm{~nm}$ field passes through a Faraday isolator to protect the SHG from back reflections and a compact Mach-Zehnder interferometer (MZ) in the green path is used to control and stabilize the pump power impinging onto the optical parametric amplifier (OPA). The photodiode $\mathrm{PD}_{\mathrm{MZ}}$ placed at one of the output ports of a 50/50 beam splitter in the green beam path is used to sense and control the MZ-output power via a servo loop. Pump power values between $1.5 \mathrm{~mW}$ and $12 \mathrm{~mW}$ can be realized by changing the offset of the MZ operating point, which determines the level of parametric (de-)amplification inside the OPA.

\subsection{Optical Parametric Amplifier}

To realize efficient cavity-enhanced parametric down-conversion, we employ a doubly resonant cavity design. The mechanical construction is similar to the SHG. The OPA cavity is again comprised of a PPKTP crystal and a piezo-actuated coupling mirror, however, the non-linear crystal has the dimensions $9.3 \mathrm{~mm} \times 2 \mathrm{~mm} \times 1 \mathrm{~mm}$. Its rear-face is polished with a $12 \mathrm{~mm}$ radius of curvature and HR coated for $1064 \mathrm{~nm}$ and $532 \mathrm{~nm}$, thus acting as the end mirror of the cavity. The intra-cavity PPKTP face is AR coated for both wavelengths. The half-inch coupling mirror has a dual wavelength coating with a designed transmissivity of around $10 \%$ at both wavelengths. A segmented active temperature stabilization scheme of the PPKTP-crystal to $\mathrm{mK}$ precision is used to simultaneously 
maintain the phase matching condition for both wavelength and the co-resonance condition of the OPA-cavity. The bulk of the crystal around the cavity waist is stabilized to the phase matching temperature with a thermoelectric heater. A short segment at the end of the crystal is set to a different temperature with a second peltier element. This region can be used to realize the cavity co-resonance condition by compensating wavelength dependent differences in the coating stacks. The generated squeezed vacuum field exits from the OPA through the coupling mirror and is separated from the pump field upon transmission through a dichroic beamsplitter (DBS). Subsequently, the squeezed field is sent through a low loss Faraday isolation stage, which protects the OPA from back-reflected light which is known to introduce excess noise at audio-band Fourier frequencies due to parasitic interferences $[4,5,17]$. Finally, an HR-mirror mounted on a motorized remote-controllable translation stage is used to either directly extract the squeezed field for downstream application or to send it for diagnostic measurements onto the balanced homodyne detector (BHD).

\subsection{Auxiliary Bright Alignment Beam}

An auxiliary bright alignment beam (BAB) is matched to the fundamental Gaussian mode of the OPA cavity and enters through the highly reflective backside of the non-linear crystal. In this configuration, the OPA cavity is highly undercoupled. On resonance it transmits approximately $0.5 \%$ of the impinging light, which corresponds to $2-3 \mathrm{~mW}$ in our setup. This "intense" field is in the same spatial mode and propagates along the same optical path as the squeezed vacuum states and can therefore be used to adjust and measure the contrast of the BHD or the alignment of the squeezed beam into the Advanced Virgo detector. Before it enters the OPA cavity, the polarization of the BAB is slightly detuned from pure s-polarization, which allows one to derive an OPA locking signal (in absence of the green pump field) on the photodiode $\mathrm{PD}_{\mathrm{BAB}}$. In this configuration of the squeezed light source, the PPKTP-crystal temperature needs to be adjusted differently in order to realize the co-resonance condition for p- and s-polarized light at $1064 \mathrm{~nm}$. Beam dumps on a remotely controllable translation stage are used to block the $532 \mathrm{~nm}$ pump beam when the BAB is needed, and vice versa, which is especially the case when squeezed vacuum states are generated.

\subsection{Coherent Control of the Squeezed Vacuum Field}

It has been shown that the generation of squeezed vacuum states of light at audio-band Fourier frequencies can be easily spoiled by light at the fundamental carrier frequency, which will introduce excess classical laser noise [5]. As a consequence, common control schemes that rely on the injection of a weak, phase modulated field at the carrier frequency into the OPA are not applicable. This problem can be overcome by the implementation of a coherent control scheme, which is based on utilizing a frequency shifted field that is phase-locked to the main carrier field [6]. We apply such a scheme to control the phase of the pump beam and the squeezing phase for homodyne detection (see below). The coherent control field is provided by Laser 2 and the frequency offset is chosen to be $7 \mathrm{MHz}$. This value is a trade-off between the signal attenuation due to the output mode cleaner stage of the Virgo detector and the residual technical noise of Laser 2. The coherent control field is mode-matched to the OPA cavity and injected through the HR back side of the PPKTP crystal. Inside the OPA the coherent control field (c.c. in Figure 2) interacts with the pump field and the reflected light detected with $\mathrm{PD}_{\text {c.c. }}$ contains information on the phase of the pump field at twice the offset frequency of the coherent control field (14 MHz). As illustrated in Figure 2, a phase shifter (piezo-driven mirror) in the pump path is used to adjust the phase of the pump field entering the OPA.

\subsection{On-Board Balanced Homodyne Detector}

An on-board balanced homodyne detector (BHD) can be used to perform characterization measurements of the generated squeezed vacuum field. The required local oscillator beam is derived from Laser 1 and is subsequently spatio-temporal filtered by a triangular mode cleaning cavity. The photodiode $\mathrm{PD}_{\mathrm{MC} 1064}$ yields an error signal to keep the mode-cleaner cavity on resonance with 
Laser 1 by means of a servo loop acting on the far mirror. The contrast of the local oscillator beam with the squeezed field on the 50/50 beamsplitter can be adjusted by employing the BAB prior to the squeezing measurements. The resulting output fields are detected with a pair of high-quantum efficiency InGaAs p-i-n photodiodes with a diameter of $500 \mu \mathrm{m}$ (PD1 and PD2 in Figure 2). Finally, the photo currents of PD1 and PD2 are subtracted from each other, amplified and then measured with a FFT analyzer (model SR785). The relative phase $\theta$ between the LO and the squeezed vacuum field can be controlled by a piezo actuated phase shifting mirror and defines the readout quadrature of the BHD. In general, the measured quadrature is $\hat{X}(\theta)=\hat{X}_{1} \cos \theta+\hat{X}_{2} \sin \theta$. A control signal for $\theta$ is derived from the beat signal generated on the BHD by the $\operatorname{dim}(\approx 1 \mu \mathrm{W})$ coherent control field (c.c.), which is transmitted through the OPA cavity and co-propagates with the squeezed vacuum field, and the bright local oscillator field. Demodulation of the detected $7 \mathrm{MHz}$ beat signal and feedback to the piezo-actuated steering mirror allows for stabilization of the BHD readout quadrature.

\subsection{Automation and Control}

For the control of the squeezed light source, we implement a hierarchical locking scheme. To this end, each analog servo control loop is equipped with additional auto-locking electronics. The individual control loop electronics are daisy-chained in order to create the desired locking sequence. Trigger signals activating the cavity control loops are derived by comparing the respective airy peak signal to appropriate pre-defined threshold voltages. As the OPA is locked via the $532 \mathrm{~nm}$ field and is resonant at $1064 \mathrm{~nm}$ only for every second green resonance, an additional trigger is derived by monitoring the non-linear gain via the pump phase coherent control error signal. Although it is not strictly necessary for the actual generation and control of the squeezed states, we have implemented additional interfaces, to extend the integration capabilities of the squeezed light source into the Advanced Virgo controls system. This enables, for example, data acquisition of all relevant channels and remote control of the servos.

\section{Results and Discussion}

Throughout the entire period of the observation run O3, the goal was to achieve a constant suppression of shot noise in the Advanced Virgo detector. A prerequisite for this is that the squeezed light source provides a constant level of squeezing. This is realized, among other things, by maintaining a constant pump power, by precisely controlling the nonlinear crystal temperature and the OPA resonance condition. We optimize these settings to obtain high-purity squeezed states at generated squeezing levels between $8-10 \mathrm{~dB}$ to keep the correlated enhancement of quantum radiation pressure noise (due to the injected anti-squeezing in the orthogonal quadrature) just below the actual remaining technical noise in the Advanced Virgo detector [16].

Figure 3 shows typical on-board measurements as taken with the diagnostic homodyne detector (BHD). All traces in this Figure are combinations of five FFT frequency windows acquired with the spectrum analyzer: $10-400 \mathrm{~Hz}, 400 \mathrm{~Hz}-1.6 \mathrm{kHz}, 1.6-6.8 \mathrm{kHz}, 6.8-25.2 \mathrm{kHz}, 25.2-102.4 \mathrm{kHz}$. Data points are averaged rms values of 200, 400, 800, 800 and 800 measurements in these frequency ranges, respectively. Trace (a) corresponds to a measurement of the unsqueezed shot noise reference, recorded with a local oscillator intensity of $2.1 \mathrm{~mW}$. Trace (b) at the bottom of the plot is a measurement of the electronic darknoise, i.e., when no light is entering the BHD. We observe a clearance between the vacuum level and the darknoise of approximately $22 \mathrm{~dB}$ down to $30 \mathrm{~Hz}$. 


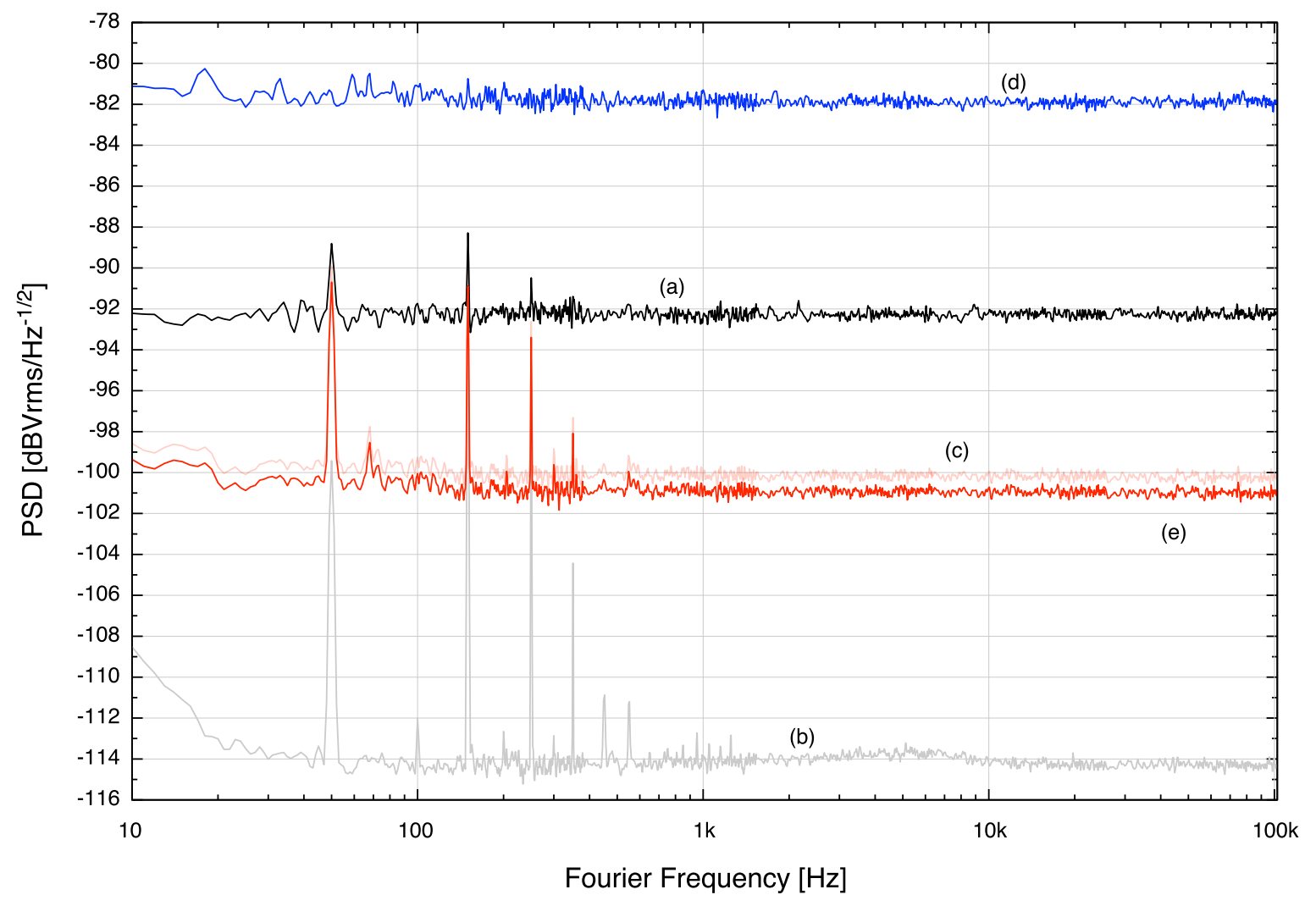

Figure 3. Noise measurements performed with the on-board diagnostic homodyne detector. All traces are combined of five FFT frequency windows acquired with the spectrum analyzer: $10-400 \mathrm{~Hz}$, $400 \mathrm{~Hz}-1.6 \mathrm{kHz}, 1.6-6.8 \mathrm{kHz}, 6.8-25.2 \mathrm{kHz}, 25.2-102.4 \mathrm{kHz}$. Data points are averaged rms values of $200,400,800,800$ and 800 measurements in these frequency ranges, respectively. Trace (a) represents the homodyne detector shot noise reference measured with a local oscillator beam of $2.1 \mathrm{~mW}$ intensity. Trace (b) is the measured electronic dark noise which is not subtracted from the data. With an OPA pump power of $6 \mathrm{~mW}$ a reduction of shot noise by up to $8.0 \mathrm{~dB}$ could be measured (trace (c)), whereas the corresponding anti-squeezing was amplified by $10.4 \mathrm{~dB}$ (trace $(\mathrm{d})$ ). By subtracting the optical loss which is introduced by the homodyne detector, one can derive an effective squeezing level of up to $9.0 \mathrm{~dB}$ that is available for the injection into the Advanced Virgo detector (trace (e)). The anti-squeezing level changes only by $0.1 \mathrm{~dB}$ due to this loss reduction and therefore increases to $10.5 \mathrm{~dB}$, indicating the high purity of the available squeezed state.

By injecting the generated squeezed states into the homodyne signal input port, the measured noise level changes according to the quantum noise variances of the squeezed or anti-squeezed states. The squeezing and anti-squeezing trace in Figure 3 were measured with a $532 \mathrm{~nm}$ pump power intensity of $6 \mathrm{~mW}$ injected into the OPA. Trace (c) shows a directly measured squeezing level of up to $8.0 \mathrm{~dB}$, with the corresponding anti-squeezing level shown in trace (d) reaching a value of approximately $10.4 \mathrm{~dB}$ above the shot noise level (trace (a)). For every squeezing and anti-squeezing measurement, the demodulation phase of the squeezing phase coherent control loop was optimized to ensure measurement of the maximum (anti-squeezing) or minimum (squeezing) quantum noise levels, respectively.

In order to deduce which squeezing level is available at the output of the squeezer box for the downstream application, one can subtract the optical loss that is introduced by the homodyne detection, which is bypassed in the case of squeezing injection into the Advanced Virgo detector. This results in a reduction of the total loss for the squeezing of about $1 \%$ due to the imperfect quantum efficiency of the photodiodes used $(\mathrm{QE}=99 \%)$ and an additional $2 \%$ due to the imperfect mode matching at the $50 / 50$ beam splitter of the homodyne detector as the measured visibility at this point was about $99 \%$. 
For this reason, one can subtract in total 3\% optical loss from the (anti-)squeezing values and obtains an effective squeezing value of $9.0 \mathrm{~dB}$ with a corresponding anti-squeezing of $10.5 \mathrm{~dB}$ available for the injection into the interferometer.

Figure 4 shows the long-term performance of the squeezed light source observed during characterization in the class 100 clean room at AEI Hannover. The data for the spectrogram are recorded in 70 consecutive squeezing spectra, each again composed of the following five FFT-windows with the respective number of averages given in parenthesis: $10-400 \mathrm{~Hz}(200), 400 \mathrm{~Hz}-1.6 \mathrm{kHz}(400), 1.6-6.8 \mathrm{kHz}$ (800), $6.8-25.2 \mathrm{kHz}$ (800), $25.2-102.4 \mathrm{kHz}$ (800). Each individual measurement takes approximately $6.5 \mathrm{~min}$, which yields a total duration of $7.8 \mathrm{~h}$. Compared to the measurements in Figure 3, a slightly higher $532 \mathrm{~nm}$ pump power was injected into the OPA, resulting in a directly measured squeezing level of up to $9.3 \mathrm{~dB}$. Such a configuration corresponds to squeezed vacuum states with more than $10 \mathrm{~dB}$ noise reduction exiting the squeezer and being available for injection into the interferometer. The horizontal lines at 50,150 and $250 \mathrm{~Hz}$ correspond to noise pick up of the electronic mains. The duty cycle of the squeezed light source for this long-term characterization was $100 \%$ and the measured squeezing level is stable within $\pm 0.1 \mathrm{~dB}$. Longer measurement campaigns were sometimes limited by temperature fluctuations of \pm 2 Kelvin in the clean room. In this case, the associated optical path length changes could not be corrected by the limited control range of the piezo-actuated phase shifting mirrors. After a lock-loss, the squeezing output is restored after no longer than $30 \mathrm{~s}$. However, since the temperature stability in the detection lab at the Advanced Virgo detector is an order of magnitude better, the problem does not arise there.

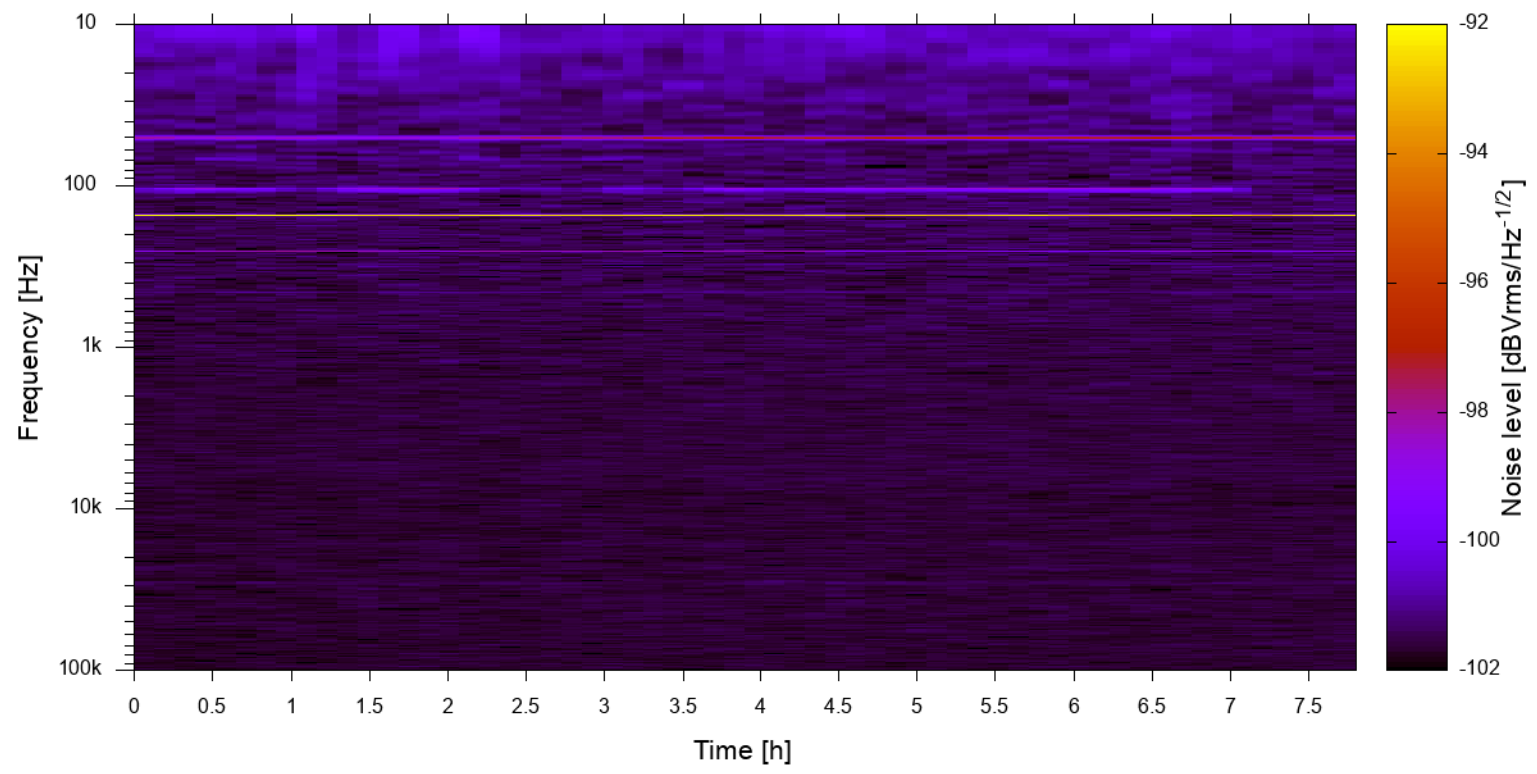

Figure 4. Spectrogram of the squeezing level in the frequency ranges from $10 \mathrm{~Hz}$ to $100 \mathrm{kHz}$. The spectrum analyzer was remotely controlled to record 70 consecutive squeezing spectra. For each measurement, the following five FFT-windows (number of averages) were chosen: $10-400 \mathrm{~Hz}(200)$, $400 \mathrm{~Hz}-1.6 \mathrm{kHz}$ (400), $1.6-6.8 \mathrm{kHz}$ (800), $6.8-25.2 \mathrm{kHz}$ (800), 25.2-102.4 kHz (800). Each measurement takes approximately $6.5 \mathrm{~min}$ yielding a total duration of $7.8 \mathrm{~h}$ accumulated squeezing data. For these measurements, the $532 \mathrm{~nm}$ pump power was slightly increased, resulting in a maximum squeezing level of $9.3 \mathrm{~dB}$. This squeezing level is stable within $\pm 0.1 \mathrm{~dB}$. A small degradation of the squeezing level is visible at frequencies below $100 \mathrm{~Hz}$. The pronounced horizontal lines correspond to spikes in the squeezing spectrum at 50,150 and $250 \mathrm{~Hz}$ due to electronic mains. 


\section{Conclusions and Outlook}

We presented a detailed description of the optical setup and electronic control of the squeezed light source as it was constructed at the AEI Hannover and later implemented at the Advanced Virgo detector to reduce the detector's quantum shot noise during the Observation Run O3. The generated squeezed states are tailored to exhibit high purity at intermediate squeezing levels $(8-10 \mathrm{~dB})$ in order to significantly reduce the interferometer shot noise level while keeping the correlated enhancement of quantum radiation pressure noise just below the actual remaining technical noise in the Advanced Virgo detector, as reported in [16]. The adjustable pump power and the associated distinct levels of applicable squeezing and anti-squeezing are a useful diagnostic tool during the commissioning of the squeezed light technology in a GW interferometer, as it allows for an analysis of the loss and phase noise budget. We have demonstrated that the squeezed light source can reliably provide squeezed vacuum states of light over the entire bandwidth of ground-based gravitational wave detectors. Therefore, this design is also suitable to reduce the quantum radiation pressure noise at the low detection frequencies of Advanced Virgo. However, in order to exploit the full potential of the squeezed light technology and simultaneously reduce quantum shot noise and quantum radiation pressure noise, the phase angle of the injected squeezing needs to be tuned as a function of the detection frequency. This frequency dependence can be realized by reflecting the generated squeezed vacuum states off a suitable filter cavity prior to the injection into the interferometer [18-20].

Author Contributions: Conceptualization, M.M. and H.V.; methodology, M.M. and H.V.; validation, M.M. and H.V.; formal analysis, M.M. and H.V.; investigation, M.M. and H.V.; data curation, M.M. and H.V.; writing-original draft preparation, M.M. and H.V.; writing-review and editing, M.M. and H.V.; visualization, M.M. and H.V. Both authors have read and agreed to the published version of the manuscript.

Funding: The authors gratefully acknowledge the support of the Max Planck Society, Leibniz Universität Hannover, and Deutsche Forschungsgemeinschaft (DFG, German Research Foundation) through Grant No. VA 1031/1-1 and Germany's Excellence Strategy Grant No. EXC-2123 QuantumFrontiers-390837967.

Conflicts of Interest: The authors declare no conflict of interest.

\section{References}

1. Caves, C.M. Quantum-mechanical noise in an interferometer. Phys. Rev. D 1981, 23, 1693-1708. [CrossRef]

2. Acernese, F.; Agathos, M.; Aiello, L.; Allocca, A.; Amato, A.; Ansoldi, S.; Antier, S.; Arène, M.; Arnaud, N.; Ascenzi, S.; et al. Increasing the Astrophysical Reach of the Advanced Virgo Detector via the Application of Squeezed Vacuum States of Light. Phys. Rev. Lett. 2019, 123, 231108. [CrossRef] [PubMed]

3. Tse, M.; Yu, H.; Kijbunchoo, N.; Fernandez-Galiana, A.; Dupej, P.; Barsotti, L.; Blair, C.D.; Brown, D.D.; Dwyer, S.E.; Effler, A.; et al. Quantum-Enhanced Advanced LIGO Detectors in the Era of Gravitational-Wave Astronomy. Phys. Rev. Lett. 2019, 123, 231107. [CrossRef] [PubMed]

4. Bowen, W.P.; Schnabel, R.; Treps, N.; Bachor, H.A.; Lam, P.K. Recovery of continuous wave squeezing at low frequencies. J. Opt. Quantum Semiclassical Opt. 2002, 4, 421-424. [CrossRef]

5. McKenzie, K.; Grosse, N.; Bowen, W.P.; Whitcomb, S.E.; Gray, M.B.; McClelland, D.E.; Lam, P.K. Squeezing in the Audio Gravitational-Wave Detection Band. Phys. Rev. Lett. 2004, 93, 161105. [CrossRef] [PubMed]

6. Vahlbruch, H.; Chelkowski, S.; Hage, B.; Franzen, A.; Danzmann, K.; Schnabel, R. Coherent Control of Vacuum Squeezing in the Gravitational-Wave Detection Band. Phys. Rev. Lett. 2006, 97, 011101. [CrossRef] [PubMed]

7. Stefszky, M.S.; Mow-Lowry, C.M.; Chua, S.S.Y.; Shaddock, D.A.; Buchler, B.C.; Vahlbruch, H.; Khalaidovski, A.; Schnabel, R.; Lam, P.K.; McClelland, D.E. Balanced homodyne detection of optical quantum states at audio-band frequencies and below. Class. Quantum Gravity 2012, 29, 145015. [CrossRef]

8. Vahlbruch, H.; Mehmet, M.; Danzmann, K.; Schnabel, R. Detection of 15 dB Squeezed States of Light and their Application for the Absolute Calibration of Photoelectric Quantum Efficiency. Phys. Rev. Lett. 2016, 117, 110801. [CrossRef] [PubMed]

9. Mehmet, M.; Vahlbruch, H. High-efficiency squeezed light generation for gravitational wave detectors. Class. Quantum Gravity 2018, 36, 015014. [CrossRef] 
10. The LIGO Scientific Collaboration. A gravitational wave observatory operating beyond the quantum shot-noise limit. Nat. Phys. 2011, 7, 962-965. [CrossRef]

11. The LIGO Scientific Collaboration. Enhanced sensitivity of the LIGO gravitational wave detector by using squeezed states of light. Nat. Photonics 2013, 7, 613-619. [CrossRef]

12. Grote, H.; Danzmann, K.; Dooley, K.L.; Schnabel, R.; Slutsky, J.; Vahlbruch, H. First Long-Term Application of Squeezed States of Light in a Gravitational-Wave Observatory. Phys. Rev. Lett. 2013, 110, 181101. [CrossRef] [PubMed]

13. Schnabel, R. Squeezed states of light and their applications in laser interferometers. Phys. Rep. 2017, 684, 1-51. [CrossRef]

14. Barsotti, L.; Harms, J.; Schnabel, R. Squeezed vacuum states of light for gravitational wave detectors. Rep. Prog. Phys. 2018, 82, 016905. [CrossRef] [PubMed]

15. Yu, H.; McCuller, L.; Tse, M.; Kijbunchoo, N.; Barsotti, L.; Mavalvala, N.; Betzwieser, J.; Blair, C.D.; Dwyer, S.E.; Effler, A.; et al. Quantum correlations between light and the kilogram-mass mirrors of LIGO. Nature 2020, 583, 43-47. [CrossRef] [PubMed]

16. Acernese, F.; Agathos, M.; Aiello, L.; Ain, A.; Allocca, A.; Amato, A.; Ansoldi, S.; Antier, S.; Arène, M.; Arnaud, N.; et al. Quantum Backaction on kg-Scale Mirrors: Observation of Radiation Pressure Noise in the Advanced Virgo Detector. Phys. Rev. Lett. 2020, 125, 131101. [CrossRef] [PubMed]

17. Vahlbruch, H.; Chelkowski, S.; Danzmann, K.; Schnabel, R. Quantum engineering of squeezed states for quantum communication and metrology. New J. Phys. 2007, 9, 371-371. [CrossRef]

18. Kimble, H.J.; Levin, Y.; Matsko, A.B.; Thorne, K.S.; Vyatchanin, S.P. Conversion of conventional gravitational-wave interferometers into quantum nondemolition interferometers by modifying their input and/or output optics. Phys. Rev. D 2001, 65, 022002. [CrossRef]

19. McCuller, L.; Whittle, C.; Ganapathy, D.; Komori, K.; Tse, M.; Fernandez-Galiana, A.; Barsotti, L.; Fritschel, P.; MacInnis, M.; Matichard, F.; et al. Frequency-Dependent Squeezing for Advanced LIGO. Phys. Rev. Lett. 2020, 124, 171102. [CrossRef] [PubMed]

20. Zhao, Y.; Aritomi, N.; Capocasa, E.; Leonardi, M.; Eisenmann, M.; Guo, Y.; Polini, E.; Tomura, A.; Arai, K.; Aso, Y.; et al. Frequency-Dependent Squeezed Vacuum Source for Broadband Quantum Noise Reduction in Advanced Gravitational-Wave Detectors. Phys. Rev. Lett. 2020, 124, 171101. [CrossRef] [PubMed]

Publisher's Note: MDPI stays neutral with regard to jurisdictional claims in published maps and institutional affiliations.

(C) 2020 by the authors. Licensee MDPI, Basel, Switzerland. This article is an open access article distributed under the terms and conditions of the Creative Commons Attribution (CC BY) license (http://creativecommons.org/licenses/by/4.0/). 